\title{
Article \\ Optimal Control Problems for Set-Valued Quasivariational Inequalities with Applications
}

\author{
Shih-Sen Chang ${ }^{1, *}$, Salahuddin ${ }^{2}$, Lin Wang ${ }^{3}{ }^{\oplus}$, Jinfang Tang ${ }^{4}$ and Liangcai Zhao ${ }^{4}$ \\ 1 Center for General Education, China Medical University, Taichung 40402, Taiwan \\ 2 Department of Mathematics, Jazan University, Jazan 45142, Saudi Arabia; dr_salah12@yahoo.com \\ 3 College of Statistics and Mathematics, Yunnan University of Fiance and Ecoomics, Kunming 650221, China; \\ w164mail@aliyun.com \\ 4 Department of mathematics, Yibin University, Yibin 644007, China; jinfangt_79@163.com (J.T.); \\ lczhaoyb@com.163.cn (L.Z.) \\ * Correspondence: changss2013@163.com
}

Citation: Chang, S.-S.; Salahuddin; Wang, L.; Tang, J.; Zhao, L. Optimal Control Problems for Set-Valued Quasivariational Inequalities with Applications. Mathematics 2022, 10, 691. https://doi.org/10.3390/ math10050691

Academic Editors: Mihai Postolache, Jen-Chih Yao and Yonghong Yao

Received: 13 January 2022

Accepted: 18 February 2022

Published: 23 February 2022

Publisher's Note: MDPI stays neutral with regard to jurisdictional claims in published maps and institutional affiliations.

Copyright: (C) 2022 by the authors. Licensee MDPI, Basel, Switzerland. This article is an open access article distributed under the terms and conditions of the Creative Commons Attribution (CC BY) license (https:// creativecommons.org/licenses/by/ $4.0 /)$.

\begin{abstract}
In this paper we investigate the optimal control problem for set-valued quasivariational inequality with unilateral constraints. Under suitable conditions, we prove that the solution to the current optimal control problem converges to a solution to old control problems. By way of application, we utilize our results presented in the paper to study the optimal control associated with boundary value problems which is described by frictional contact problems and a stationary heat transfer problem with unilateral constraints.
\end{abstract}

Keywords: convergence results; inverse strong monotonicity; frictional contact; heat transfer; optimal control; optimal pair; set-valued quasivariational inequality problem; unilateral constraint

MSC: 49J27; 49K20; 74M15; 74M10; 49J40; 47H09; 47J20; 54H25; 49J53; 58E35; 35J66

\section{Introduction}

The theory of variational techniques refers to the tool for estimating the appropriate auxiliary function that attains a minimum. This can be conceived as a mathematical model of the concept of the least action in physics and engineering. Since so many key results in mathematics, particularly in the analysis, have their origins in the physical sciences, it is entirely natural that they can be associated in one way or another to variational techniques. The use of variational concepts in mathematical proofs has a long history. This can be traced back to Johann Bernoulli's problem of the Brachistochrone and its solutions leading to the development of the calculus of variations. Since then the method has been used in a variety of fields of mathematics, see [1-8].

An important feature of the new variational techniques is that they are able to handle nonsmooth functions, sets and set-valued functions reasonably equally well. The role of nonsmooth, lower semicontinuous functions and associated subdifferential functions is frequently extended. Mordukhovich [9] begins with variational geometry on closed sets and then determines the epigraph and graphs of functions and set-valued functions. Setvalued functions arise naturally in many situations. Some frequently encountered examples are the level sets and sublevel sets of a function, various subdifferentials of nonsmooth functions, the solution sets of an optimization problem depending on some parameters and the vector field of a variational system.

The theory of optimal control problem for variational inequalities is an expanding and vibrant field of applied mathematics that has various applications. Most of the models in physics, mechanics, engineering science, elasticity and contact problems are expressed in terms of strongly nonlinear boundary value problems with partial differential equations which, in a weak formulation, lead to variational inequalities. Results in the study of optimal control for variational inequalities have been addressed in several research papers, 
including [10-16] and the applications in mechanics, see [17-19]. These are some more references of optimal control in mechanics, physics and engineering where we can see the discussion, see [20-25].

In this paper we consider the optimal control problem for a general class of set-valued quasivariational inequality problems with unilateral constraints. Under the appropriate data assumptions, we prove that the solution of the current optimal control problem converges to a solution of previous control problems. As an application, we deploy our results to demonstrate the frictional contact of an elastic material with a rigid-deformable foundation and a stationary heat transfer boundary value problem with unilateral constraints.

\section{Preliminaries}

Throughout the text " $\rightarrow$ " and " $\rightarrow$ " stand for the weak and the strong convergence, respectively.

Let $X$ and $Y$ be real Hilbert spaces endowed with the inner products $\langle\cdot, \cdot\rangle_{X}$ and $\langle\cdot, \cdot\rangle_{Y}$ respectively. We denote by $\|\cdot\|_{X},\|\cdot\|_{Y}$ the norms on the spaces $X$ and $Y$, respectively. Let $2^{X}$ be the family of all the nonempty subsets of $X$ and $C B(X)$ be the family of all the nonempty closed bounded subsets of $X$. Assume that $\Omega \subset X, \mathcal{N}: X \longrightarrow X, J: X \times X \longrightarrow \mathbb{R}$, $\varsigma: X \longrightarrow Y, f \in Y$ and $\mathcal{T}: \Omega \longrightarrow 2^{X}$ is the set-valued operator. Then, we consider the following set-valued quasi-variational inequality problem for finding $x \in \Omega, u \in \mathcal{T}(x)$ such that

$$
\langle\mathcal{N}(u), y-x\rangle_{X}+j(x, y)-j(x, x) \geq\langle f, \varsigma y-\varsigma x\rangle_{Y}, \forall y \in \Omega,
$$

The set of admissible pairs for inequality (1) is given by

$$
\mathcal{U}_{a d}=\{(x, f) \in \Omega \times Y \text { such that (1) holds }\}
$$

and we consider a cost functional $£: X \times Y \longrightarrow \mathbb{R}$. Then, the optimal control problem for finding $\left(x^{\star}, f^{\star}\right) \in \mathcal{U}_{a d}$ such that

$$
£\left(x^{\star}, f^{\star}\right)=\min _{(x, f) \in \mathcal{U}_{a d}} £(x, f) .
$$

Let $\tilde{\Omega} \subset X$ be a set, $\tilde{\mathcal{N}}: X \longrightarrow X$ an operator, $\tilde{\mathcal{T}}: \Omega \longrightarrow 2^{X}$ be a set-valued operator and an element $\tilde{f} \in Y$. With these data, we suggest the following perturbation of the problem (1): for finding $\tilde{x} \in \tilde{\Omega}, \tilde{u} \in \tilde{\mathcal{T}}(\tilde{x})$ such that

$$
\langle\tilde{\mathcal{N}}(\tilde{u}), y-\tilde{x}\rangle_{X}+j(\tilde{x}, y)-j(\tilde{x}, \tilde{x}) \geq\langle\tilde{f}, \varsigma y-\varsigma \tilde{x}\rangle_{Y}, \forall y \in \tilde{\Omega} .
$$

We associate to problem (4), the admissible pairs set defined by

$$
\tilde{\mathcal{U}}_{a d}=\{(\tilde{x}, \tilde{f}) \in \tilde{\Omega} \times Y \text { such that (4) holds }\}
$$

and, for a cost functional $\tilde{E}: X \times Y \longrightarrow \mathbb{R}$, we construct the following perturbation of the optimal control problem (3) for finding $\left(\tilde{x}^{\star}, \tilde{f}^{\star}\right) \in \tilde{\mathcal{U}}_{a d}$ such that

$$
\tilde{E}\left(\tilde{x}^{\star}, \tilde{f}^{\star}\right)=\min _{(\tilde{x}, \tilde{f}) \in \tilde{\mathcal{U}}_{a d}} \tilde{E}(\tilde{x}, \tilde{f}) .
$$

In our study of (1) we consider the following assumptions:

(i)

$\Omega$ is a nonempty, closed, convex subset of $X$.

(ii) $\mathcal{N}$ is inverse strongly monotone, if there exist $\alpha_{\mathcal{N}}>0$ such that

$$
\langle\mathcal{N}(u)-\mathcal{N}(v), x-y\rangle_{X} \geq \alpha_{\mathcal{N}}\|\mathcal{N}(u)-\mathcal{N}(v)\|_{X}^{2}, \forall x, y \in X, u \in \mathcal{T}(x), v \in \mathcal{T}(y) .
$$


(iii) $\mathcal{T}$ is $\mathcal{H}$-Hausdorff Lipschitz continuous then there exist $\alpha_{\mathcal{T}} \geq 0$ such that

$$
\|u-v\|_{X} \leq \mathcal{H}(\mathcal{T}(x), \mathcal{T}(y))_{X} \leq \alpha_{\mathcal{T}}\|x-y\|_{X}, \forall x, y \in X,
$$

where $\mathcal{H}(\cdot, \cdot)$ is the Hausdorff metric on $C B(X)$.

(iv) $\mathcal{N}$ is Lipschitz continuous if there exist constants $\beta_{\mathcal{N}}>0$ such that

$$
\|\mathcal{N}(x)-\mathcal{N}(y)\| \leq \beta_{\mathcal{N}}\|x-y\|_{X}, \forall x, y \in X
$$

(v) For all $\eta \in X$,

$$
\jmath(\eta, \cdot): X \longrightarrow \mathbb{R} \text { is convex and lower semicontinuous (l.s.c), }
$$

(vi) There exists $\alpha_{\jmath} \geq 0$ such that

$$
\begin{aligned}
\jmath\left(\eta_{1}, y_{2}\right)-\jmath\left(\eta_{1}, y_{1}\right) & +\jmath\left(\eta_{2}, y_{1}\right)-\jmath\left(\eta_{2}, y_{2}\right) \\
& \leq \alpha_{\jmath}\left\|\eta_{1}-\eta_{2}\right\|_{X}\left\|y_{1}-y_{2}\right\|_{X}, \forall \eta_{1}, \eta_{2}, y_{1}, y_{2} \in X
\end{aligned}
$$

(vii)

$$
\alpha_{\mathcal{N}} \beta_{\mathcal{N}}^{2} \alpha_{\mathcal{T}}^{2}>\alpha_{\jmath}
$$

(viii)

$$
f \in Y \text {. }
$$

(ix) $\varsigma$ is a linear continuous operator, i.e., there exists $\pi_{0}>0$ such that

$$
\|\varsigma y\|_{Y} \leq \pi_{0}\|y\|_{X}, \forall y \in X
$$

Theorem 1 ([26]). Assume that (7)-(15) satisfied. Then the set-valued quasi variational inequality (1) has a unique solution.

In the study of the optimal control problem, we assume that

$$
£(x, f)=\mathcal{P}(x)+\mathcal{D}(f), \forall x \in X, f \in Y,
$$

where $\mathcal{P}: X \longrightarrow \mathbb{R}$ is continuous, positive and bounded, i.e.,

$$
\left\{\begin{array}{l}
\text { (a) } y_{\ell} \longrightarrow y \in X \Rightarrow \mathcal{P}\left(y_{\ell}\right) \longrightarrow \mathcal{P}(y) \\
\text { (b) } \mathcal{P}(y) \geq 0, \forall y \in X \\
\text { (c) } \mathcal{P} \text { maps bounded sets in } X \text { into bounded sets in } \mathbb{R} .
\end{array}\right.
$$

and $\mathcal{D}: Y \longrightarrow \mathbb{R}$ is weakly lower semicontinuous and coercive, i.e.,

$$
\left\{\begin{array}{l}
(a) f_{\ell} \rightarrow f \in Y \Rightarrow \liminf \mathcal{D}\left(f_{\ell}\right) \geq \mathcal{D}(f), \\
(b)\left\|f_{\ell}\right\|_{Y} \rightarrow \infty \Rightarrow \mathcal{D}\left(f_{\ell}\right) \rightarrow \infty
\end{array}\right.
$$

Also there exist $\rho \geq 0, \varrho \geq 0$ such that

$$
\begin{gathered}
\jmath\left(\eta, y_{1}\right)-\jmath\left(\eta, y_{2}\right) \leq\left(\rho+\varrho\|\eta\|_{X}\right)\left\|y_{1}-y_{2}\right\|_{X}, \forall \eta, y_{1}, y_{2} \in X, \\
\alpha_{\mathcal{N}} \beta_{\mathcal{N}}^{2} \alpha_{\mathcal{T}}^{2}>\varrho .
\end{gathered}
$$

For any sequences $\left\{\eta_{\ell}\right\} \subset X,\left\{x_{\ell}\right\} \subset X$ such that

$$
\left\{\begin{array}{l}
\eta_{\ell} \rightarrow \eta \in X, x_{\ell} \rightarrow x \in X \text { one has } \\
\lim \sup \left(\jmath\left(\eta_{\ell}, y\right)-\jmath\left(\eta_{\ell}, x_{\ell}\right)\right) \leq \jmath(\eta, y)-\jmath(\eta, x), \quad \forall y \in X .
\end{array}\right.
$$


For any sequence $\left\{y_{\ell}\right\} \subset X$ such that

$$
y_{\ell} \rightarrow y \in X \text { one has } s y_{\ell} \rightarrow \varsigma y \in Y .
$$

Theorem 2 ([26]). Assume that (7)-(22) satisfied. Then at least one solution

$$
\left(x^{\star}, f^{\star}\right) \in \mathcal{U}_{a d}
$$

of the problem (3) exists.

\section{Convergence Analysis}

In this section, we state and prove the convergence result for the solution of problem (4), in this case when the problem has a particular structure. To do this, we consider two sequences $\left\{\gamma_{\ell}\right\} \subset \mathbb{R},\left\{f_{\ell}\right\} \subset Y$ and an operator $\mathcal{M}: X \longrightarrow X$. For $\ell \in \mathbb{N}$, let $\mathcal{N}_{\ell}: X \longrightarrow X$ be the operator and $\mathcal{T}_{\ell}: \Omega \longrightarrow 2^{X}$ be the set-valued operator defined by

$$
\mathcal{N}_{\ell}(u)=\mathcal{N}(u)+\frac{1}{\gamma_{\ell}} \mathcal{M}(u), \quad \forall x \in X, u \in \mathcal{T}(x) .
$$

Suppose that if $\tilde{\mathcal{N}}=\mathcal{N}_{\ell}$ and $\tilde{f}=f_{\ell}$, then we have a problem for finding $x_{\ell} \in \tilde{\Omega}, u_{\ell} \in \mathcal{T}\left(x_{\ell}\right)$ such that

$$
\begin{aligned}
\left\langle\mathcal{N}\left(u_{\ell}\right), y-x_{\ell}\right\rangle_{X}+\frac{1}{\gamma_{\ell}}\left\langle\mathcal{M}\left(u_{\ell}\right), y-x_{\ell}\right\rangle_{X} & +\jmath\left(x_{\ell}, y\right)-\jmath\left(x_{\ell}, x_{\ell}\right) \\
& \geq\left\langle f_{\ell}, \varsigma y-\varsigma x_{\ell}\right\rangle_{Y}, \forall y \in \tilde{\Omega} .
\end{aligned}
$$

Remark 1. $\tilde{\Omega}=X$ then (24) denotes the penalty problem of (1), under the suitable assumptions of $\mathcal{M}$, see $[4,27]$.

To prove the unique solution of (24), we use the following assumptions.

$\tilde{\Omega}$ is a nonempty, closed, convex subset of $X$.

$\mathcal{M}: X \longrightarrow X$ is an inverse strongly monotone, and Lipschitz continuous operator.

$$
\begin{aligned}
& \gamma_{\ell}>0, \quad \forall \ell \in \mathbb{N} . \\
& f_{\ell} \in Y, \quad \forall \ell \in \mathbb{N} .
\end{aligned}
$$

Lemma 1 ([28]). Assume that (8)-(13), (15), (25)-(28) satisfied. Then for each $\ell \in \mathbb{N}$, there exists a unique solution $x_{\ell} \in X, u_{\ell} \in \mathcal{T}\left(x_{\ell}\right)$ to the problem (24).

To show the behaviour of the solution of the problem (24) as $\ell \longrightarrow \infty$. We consider the following additional assumptions:

$$
\begin{gathered}
\gamma_{\ell} \longrightarrow 0 \text { as } \ell \longrightarrow \infty . \\
f_{\ell} \rightarrow f \in Y \text { as } \ell \longrightarrow \infty . \\
\Omega \subset \tilde{\Omega} . \\
\left\{\begin{array}{l}
(a)\langle\mathcal{M}(u), y-x\rangle_{X}, \forall x \in \tilde{\Omega}, y \in \Omega, u \in \mathcal{T}(x), \\
(b) x \in \tilde{\Omega}, u \in \tilde{\mathcal{T}}(x),\langle\mathcal{M}(u), y-x\rangle_{X}=0, \forall y \in \Omega \Rightarrow x \in \Omega, u \in \mathcal{T}(x) .
\end{array}\right.
\end{gathered}
$$

Remark 2. When $\tilde{\Omega}=X$, condition (32) is satisfied for any penalty operator of the set $\Omega$, see $[29,30]$. 
Theorem 3. Assume that (7)-(15), (19)-(22), (25)-(32) and, for each $\ell \in \mathbb{N}$, denote by $x_{\ell}$ and $u_{\ell}$ the solution of (24). Then

$$
\begin{aligned}
& u_{\ell} \longrightarrow u \in \mathcal{T}(x) \subset X, \text { as } \ell \longrightarrow \infty, \\
& x_{\ell} \longrightarrow x \in X, \text { as } \ell \longrightarrow \infty,
\end{aligned}
$$

where $x$ and $u$ are solutions of problem (1).

Proof. The proof of Theorem 3 consists of several steps.

(i) The weak convergence result.

We assert that there is an element $\tilde{x} \in \tilde{\Omega}$ and a subsequence of $\left\{x_{\ell}\right\}$, which is still denoted by $\left\{x_{\ell}\right\}$, such that

$$
x_{\ell} \rightarrow \tilde{x} \in X \text { as } \ell \longrightarrow \infty \text {. }
$$

Similarly we have $\tilde{u} \in \tilde{\mathcal{T}}(\tilde{x})$ and a subsequence $\left\{u_{\ell}\right\}$ such that

$$
u_{\ell} \rightarrow u \in \mathcal{T}(x) \subset X \text { as } \ell \longrightarrow \infty
$$

To prove the claim, we establish the boundedness of the sequence $\left\{x_{\ell}\right\}$ in $X$. Let $\ell \in \mathbb{N}$. We make use of the assumption (31) and take $y=x$ in (24) to see that

$$
\left\langle\mathcal{N}\left(u_{\ell}\right), x_{\ell}-x\right\rangle_{X} \leq \frac{1}{\gamma_{\ell}}\left\langle\mathcal{M}\left(u_{\ell}\right), x-x_{\ell}\right\rangle_{X}+\jmath\left(x_{\ell}, x\right)-\jmath\left(x_{\ell}, x_{\ell}\right)+\left\langle f_{\ell}, \varsigma x_{\ell}-\varsigma x\right\rangle_{Y} .
$$

Since $\mathcal{N}$ is inversely strongly monotone and Lipschitz continuous, and $\mathcal{T}$ is $\mathcal{H}$ Hausdorff Lipschitz continuous, we have

$$
\begin{aligned}
\left(\alpha_{\mathcal{N}} \beta_{\mathcal{N}^{\mathcal{T}}}^{2} \alpha_{\mathcal{T}}^{2}\right)\left\|x_{\ell}-x\right\|_{X}^{2} & \leq\left\langle\mathcal{N}(u), x-x_{\ell}\right\rangle_{X}+\frac{1}{\gamma_{\ell}}\left\langle\mathcal{M}\left(u_{\ell}\right), x-x_{\ell}\right\rangle_{X}+\jmath\left(x_{\ell}, x\right) \\
& -\jmath\left(x_{\ell}, x_{\ell}\right)+\left\langle f_{\ell}, \varsigma x_{\ell}-\varsigma x\right\rangle_{Y} .
\end{aligned}
$$

Next, assumption (32) implies that

$$
\left\langle\mathcal{M}\left(u_{\ell}\right), x-x_{\ell}\right\rangle_{X} \leq 0
$$

and assumptions (11)-(12), (19) yield

$$
\begin{aligned}
\jmath\left(x_{\ell}, x\right)-\jmath\left(x_{\ell}, x_{\ell}\right) & =\left(\jmath\left(x_{\ell}, x\right)-\jmath\left(x_{\ell}, x_{\ell}\right)+\jmath\left(x, x_{\ell}\right)-\jmath(x, x)\right)+\left(\jmath(x, x)-\jmath\left(x, x_{\ell}\right)\right) \\
& \leq \alpha_{\jmath}\left\|x_{\ell}-x\right\|_{X}^{2}+\left(\rho+\varrho\|x\|_{X}\right)\left\|x_{\ell}-x\right\|_{X}
\end{aligned}
$$

On the other side, using (15) we find that

$$
\left\langle\mathcal{N}(u), x-x_{\ell}\right\rangle_{X}+\left\langle f_{\ell}, \varsigma x_{\ell}-\varsigma x\right\rangle_{Y} \leq\left(\|\mathcal{N}(u)\|_{X}+\pi_{0}\left\|f_{\ell}\right\|_{Y}\right)\left\|x_{\ell}-x\right\|_{X}
$$

Now adding inequalities (33)-(36), we have

$$
\begin{aligned}
\left(\alpha_{\mathcal{N}} \beta_{\mathcal{N}}^{2} \alpha_{\mathcal{T}}^{2}\right)\left\|x_{\ell}-x\right\|_{X}^{2} & \leq\left(\|\mathcal{N}(u)\|_{X}+\pi_{0}\left\|f_{\ell}\right\|_{Y}\right)\left\|x_{\ell}-x\right\|_{X}+\alpha_{\jmath}\left\|x_{\ell}-x\right\|_{X}^{2} \\
& +\left(\rho+\varrho\|x\|_{X}\right)\left\|x_{\ell}-x\right\|_{X} .
\end{aligned}
$$

From (30) we see that the sequence $\left\{f_{\ell}\right\}$ is bounded in $Y$. Therefore, using inequality (37) and the smallness assumption (13), we deduce that there exists a constant $\chi>0$ independent of $\ell$ such that

$$
\left\|x_{\ell}-x\right\|_{X} \leq \chi
$$


This implies that the sequence $\left\{x_{\ell}\right\}$ is bounded in $X$. Thus, from the reflexivity of $X$, by passing to a subsequence, if necessary, we have

$$
x_{\ell} \rightarrow \tilde{x} \in X \text {, as } \ell \longrightarrow \infty \text {, with some } \tilde{x} \in X \text {. }
$$

Moreover, assumption (25) and (38) implies that

$$
\tilde{x} \in \tilde{\Omega} \text {. }
$$

Again, since

$$
\left\|u_{\ell}-u\right\|_{X} \leq \mathcal{H}\left(\mathcal{T}\left(x_{\ell}\right), \mathcal{T}(x)\right)_{X} \leq \alpha_{\mathcal{T}}\left\|x_{\ell}-x\right\|_{X} \leq \chi
$$

implies that

$$
\left\|u_{\ell}-u\right\|_{X} \leq \chi
$$

Therefore, the sequence $\left\{u_{\ell}\right\}$ is also bounded in $X$. Thus, from (38) we have

$$
u_{\ell} \rightarrow \tilde{u} \in \mathcal{T}(\tilde{x}) \subset X, \text { as } \ell \longrightarrow \infty
$$

to show that

$$
\tilde{u} \in \tilde{\Omega}
$$

and the proof of assertion is completed.

(ii) The behaviour of weak limit.

Next, we show that $\tilde{x}$ is a solution to (1).

Let $y$ be a given element in $\tilde{\Omega}$ and let $\ell \in \mathbb{N}$. We use (24) to obtain that

$$
\begin{aligned}
\frac{1}{\gamma_{\ell}}\left\langle\mathcal{M}\left(u_{\ell}\right), x_{\ell}-y\right\rangle_{X} \leq & \left\langle\mathcal{N}\left(u_{\ell}\right), y-x_{\ell}\right\rangle_{X}+\jmath\left(x_{\ell}, y\right)-\jmath\left(x_{\ell}, x_{\ell}\right) \\
& +\left\langle f_{\ell}, \varsigma x_{\ell}-\varsigma y\right\rangle_{Y}, \forall u_{\ell} \in \mathcal{T}\left(x_{\ell}\right) .
\end{aligned}
$$

Then, by conditions (8)-(10), (30), (19), (15), using the boundedness of the sequences $\left\{x_{\ell}\right\}$ and $\left\{u_{\ell}\right\}$, we deduce that each term in the right hand side of inequality (39) is bounded. This means that there exists a constant $\lambda>0$ which does not depend on $\ell$ such that

$$
\left\langle\mathcal{M}\left(u_{\ell}\right), x_{\ell}-y\right\rangle_{X} \leq \gamma_{\ell} \lambda, \forall u_{\ell} \in \mathcal{T}\left(x_{\ell}\right) .
$$

We now pass to the upper limit of this inequality and apply the convergence (29) to derive that

$$
\limsup \left\langle\mathcal{M}\left(u_{\ell}\right), x_{\ell}-y\right\rangle_{X} \leq 0, \forall u_{\ell} \in \mathcal{T}\left(x_{\ell}\right) .
$$

Next, we take $y=\tilde{x}$ in (40) and find that

$$
\lim \sup \left\langle\mathcal{M}\left(u_{\ell}\right), x_{\ell}-\tilde{x}\right\rangle_{X} \leq 0, \forall u_{\ell} \in \mathcal{T}\left(x_{\ell}\right)
$$

Therefore, using assumption (26) and a standard pseudomonotonicity argument (Proposition 1.23 in $[27,31]$ ) we obtain that

$$
\liminf \left\langle\mathcal{M}\left(u_{\ell}\right), x_{\ell}-y\right\rangle_{X} \geq\langle\mathcal{M}(\tilde{u}), \tilde{x}-y\rangle_{X}, \forall y \in X, u_{\ell} \in \mathcal{T}\left(x_{\ell}\right), \tilde{u}_{\ell} \in \mathcal{T}\left(\tilde{x}_{\ell}\right) .
$$

We now combine the inequalities (42) and (40) to get

$$
\langle\mathcal{M}(\tilde{u}), \tilde{x}-y\rangle_{X} \leq 0, \forall y \in \tilde{\Omega}, \tilde{u} \in \mathcal{T}(\tilde{x}) .
$$

Using the assumption (32)(b), we can derive that $\tilde{x} \in \tilde{\Omega}, \tilde{u} \in \mathcal{T}(\tilde{x})$. 
Now, consider an element $y \in \tilde{\Omega}$. We use (31) and (24) to get

$$
\begin{aligned}
\left\langle\mathcal{N}\left(u_{\ell}\right), x_{\ell}-y\right\rangle_{X} \leq & \frac{1}{\gamma_{\ell}}\left\langle\mathcal{M}\left(u_{\ell}\right), y-x_{\ell}\right\rangle_{X}+\jmath\left(x_{\ell}, y\right)-\jmath\left(x_{\ell}, x_{\ell}\right) \\
& +\left\langle f_{\ell}, \varsigma x_{\ell}-\varsigma y\right\rangle_{Y}, \forall u_{\ell} \in \mathcal{T}\left(x_{\ell}\right) .
\end{aligned}
$$

Therefore, by using the assumption (32)(a) to get

$$
\left\langle\mathcal{N}\left(u_{\ell}\right), x_{\ell}-y\right\rangle_{X} \leq \jmath\left(x_{\ell}, y\right)-\jmath\left(x_{\ell}, x_{\ell}\right)+\left\langle f_{\ell}, \varsigma x_{\ell}-\varsigma y\right\rangle_{Y}, \forall u_{\ell} \in \mathcal{T}\left(x_{\ell}\right) .
$$

Next, using (38) and assumption (21) we have

$$
\limsup \left(\jmath\left(x_{\ell}, y\right)-\jmath\left(x_{\ell}, x_{\ell}\right)\right) \leq \jmath(\tilde{x}, y)-\jmath(\tilde{x}, \tilde{x}) .
$$

On the other side, assumption (30), (22) and the convergence (38) yield

$$
\left\langle f_{\ell}, \varsigma x_{\ell}-\varsigma y\right\rangle_{X} \longrightarrow\langle f, \varsigma \tilde{x}-\varsigma y\rangle_{Y}
$$

Using the relations (43)-(45) to see that

$$
\lim \sup \left\langle\mathcal{N}\left(u_{\ell}\right), x_{\ell}-y\right\rangle_{X} \leq \jmath(\tilde{x}, y)-\jmath(\tilde{x}, \tilde{x})+\langle f, \varsigma \tilde{x}-\varsigma y\rangle_{X}, \forall u_{\ell} \in \mathcal{T}\left(x_{\ell}\right) .
$$

Now, taking $y=\tilde{x}$ in (46) we get

$$
\lim \sup \left\langle\mathcal{N}\left(u_{\ell}\right), x_{\ell}-\tilde{x}\right\rangle_{X} \leq 0, \forall u_{\ell} \in \mathcal{T}\left(x_{\ell}\right) .
$$

This inequality together with (38) and the pseudomonotonicity of $\mathcal{N}$ implies that

$$
\langle\mathcal{N}(\tilde{u}), \tilde{x}-y\rangle_{X} \leq \liminf \left\langle\mathcal{N}\left(u_{\ell}\right), x_{\ell}-y\right\rangle_{X}, \forall y \in X, \tilde{u} \in \mathcal{T}(\tilde{x}) .
$$

Combining (48) and (46), we have

$$
\langle\mathcal{N}(\tilde{u}), \tilde{x}-y\rangle_{X} \leq \jmath(\tilde{x}, y)-\jmath(\tilde{x}, \tilde{x})+\langle f, \varsigma \tilde{x}-\varsigma y\rangle_{Y}, y \in \Omega, \tilde{u} \in \mathcal{T}(\tilde{x}) .
$$

Hence, it follows that $\tilde{x} \in \Omega$ and $\tilde{u} \in \mathcal{T}(\tilde{x})$ is a solution to (1), as claimed.

(iii) The second weak convergence result: Here we prove that the whole sequences $\left\{x_{\ell}\right\}$ and $\left\{u_{\ell}\right\}$ are weakly convergent.

Since the problem (1) has a unique solution $x \in \Omega$. Then we derive from the previous step that $\tilde{x}=x$. Furthermore, a comprehensive analysis of the proof in step (ii) establishes that the every subsequence of $\left\{x_{\ell}\right\}$ which converges weakly in $X$ has the weak limit point $x$. In addition, we recall that the sequence $\left\{x_{\ell}\right\}$ is bounded in $X$. Therefore, using a standard argument, we may derive that the whole sequence $\left\{x_{\ell}\right\}$ converges weakly in $X$ to $x$, as $\ell \longrightarrow \infty$.

Now, from the $\mathcal{H}$-Hausdorff Lipschitz continuity of $\mathcal{T}$ and (38), we note that the sequence $\left\{u_{\ell}\right\}$ is bounded in $X$ and i.e.,

$$
u_{\ell} \rightarrow u \in \mathcal{T}(x) \subset X, \text { as } \ell \rightarrow \infty
$$

(iv) Strong convergence. In the final step of the proof, we prove that

$$
x_{\ell} \longrightarrow x \in X, \text { as } \ell \longrightarrow \infty \text {. }
$$

We take $y=\tilde{x} \in \Omega$ in (48) and use (47) to obtain

$$
\begin{aligned}
0 & \leq \liminf \left\langle\mathcal{N}\left(u_{\ell}\right), x_{\ell}-\tilde{x}\right\rangle_{X} \\
& \leq \lim \sup \left\langle\mathcal{N}\left(u_{\ell}\right), x_{\ell}-\tilde{x}\right\rangle_{X} \\
& \leq 0, \quad \forall u_{\ell} \in \mathcal{T}\left(x_{\ell}\right),
\end{aligned}
$$


which shows that

$$
\left\langle\mathcal{N}\left(u_{\ell}\right), x_{\ell}-\tilde{x}\right\rangle_{X} \longrightarrow 0 \text { as } \ell \longrightarrow \infty \text {. }
$$

Again, since

$$
\left\|u_{\ell}-u\right\|_{X} \leq \mathcal{H}\left(\mathcal{T}\left(x_{\ell}\right), \mathcal{T}(x)\right)_{X} \leq \alpha_{\mathcal{T}}\left\|x_{\ell}-x\right\|_{X} \longrightarrow 0 \text { as } \ell \longrightarrow \infty .
$$

Therefore, using inequality $\tilde{x}=x$, the inversely strong monotonicity and Lipschitz continuity of the operator $\mathcal{N}$, the $\mathcal{H}$-Hausdorff Lipschitz continuity of the set-valued operator $\mathcal{T}$ and the convergence of $x_{\ell} \rightarrow x \in X$, and $u_{\ell} \rightarrow u \in \mathcal{T}(x) \subset X$, we have

$$
\begin{aligned}
\left(\alpha_{\mathcal{N}} \beta_{\mathcal{N}}^{2} \alpha_{\mathcal{T}}^{2}\right)\left\|x_{\ell}-x\right\|_{X}^{2} & \leq\left\langle\mathcal{N}\left(u_{\ell}\right)-\mathcal{N}(u), x_{\ell}-x\right\rangle_{X} \\
& =\left\langle\mathcal{N}\left(u_{\ell}\right), x_{\ell}-x\right\rangle_{X}-\left\langle\mathcal{N}(u), x_{\ell}-x\right\rangle_{X} \longrightarrow 0 \text { as } \ell \longrightarrow \infty .
\end{aligned}
$$

Hence, it follows that

$$
x_{\ell} \longrightarrow x \in X \text {, and } u_{\ell} \longrightarrow u \in \mathcal{T}(x) \subset X,
$$

and proof is completed.

\section{Convergence Theory of Optimal Pairs}

In this section, we prove the convergence solution for an optimal control problem (24). To this end, we preserve the notations and assumptions in the previous section, and we define the set of admissible pairings for (24) by

$$
\mathcal{U}_{a d}^{\ell}=\left\{\left(x_{\ell}, f_{\ell}\right) \in \tilde{\Omega} \times Y \text { such that (24) holds }\right\} .
$$

Then, the optimal control problem corresponding to (24) is: For finding $\left(x_{\ell}^{\star}, f_{\ell}^{\star}\right) \in \mathcal{U}_{a d}^{\ell}$ such that

$$
£_{\ell}\left(x_{\ell}^{\star}, f_{\ell}^{\star}\right)=\min _{\left(x_{\ell}, f_{\ell}\right) \in \mathcal{U}_{a d}^{\ell}} £_{\ell}\left(x_{\ell}, f_{\ell}\right) .
$$

In the study of (51), we assume that

$$
£_{\ell}(x, f)=\mathcal{P}_{\ell}(x)+\mathcal{D}_{\ell}(f), \forall x \in X, f \in Y,
$$

where $\mathcal{P}_{\ell}$ and $\mathcal{D}_{\ell}$ are functions which satisfy assumptions (17) and (18), for each $\ell \in \mathbb{N}$.

We note that when we use these assumptions for the functions $\mathcal{P}_{\ell}$ and $\mathcal{D}_{\ell}$, we refer to $(17)_{\ell}$ and $(18)_{\ell}$, respectively. Using the Theorem 2 we have the following result.

Lemma 2 ([28]). Assume that (8)-(13), (15), (52), (17) $\ell,(18)_{\ell},(19)-(22)$ and (25)-(28) hold. Then, for each $\ell \in \mathbb{N}$, there exists at least one solution $\left(x_{\ell}^{\star}, f_{\ell}^{\star}\right) \in \mathcal{U}_{a d}^{\ell}$ of the problem (51).

To study the behavior of the sequence of solutions of (51) as $\ell \longrightarrow \infty$, we consider the following additional hypotheses:

$$
\begin{gathered}
x_{\ell} \rightarrow x \in X \Rightarrow \mathcal{P}_{\ell}\left(x_{\ell}\right) \rightarrow \mathcal{P}(x) . \\
f_{\ell} \rightarrow f \in Y \Rightarrow \liminf \mathcal{D}_{\ell}\left(f_{\ell}\right) \geq \mathcal{D}(f) . \\
\left\|f_{\ell}\right\|_{Y} \longrightarrow \infty \Rightarrow \mathcal{D}_{\ell}\left(f_{\ell}\right) \rightarrow \infty . \\
\mathcal{D}_{\ell}(f) \rightarrow \mathcal{D}(f), \forall f \in Y .
\end{gathered}
$$

Theorem 4. Assume that (7)-(13), (15), (22), (25)-(29), (31)-(32), (17) $\ell_{\ell}(18)_{\ell}$ and (52)-(56) hold. Assume that $\left\{\left(x_{\ell}^{\star}, f_{\ell}^{\star}\right)\right\}$ is a sequence of solutions of the problem (51) then there exists a subsequence 
of the sequence $\left\{\left(x_{\ell}^{\star}, f_{\ell}^{\star}\right)\right\}$, again denoted by $\left\{\left(x_{\ell}^{\star}, f_{\ell}^{\star}\right)\right\}$, and an element $\left(x^{\star}, f^{\star}\right) \in X \times Y$ such that

$$
\begin{aligned}
& f_{\ell}^{\star} \rightarrow f^{\star} \in Y \text { as } \ell \longrightarrow \infty, \\
& x_{\ell}^{\star} \rightarrow x^{\star} \in X \text { as } \ell \longrightarrow \infty, \\
& \left(x^{\star}, f^{\star}\right) \text { is a solution of (3) }
\end{aligned}
$$

Proof. The proof is carried out in following manner.

(i) Boundedness analysis. We claim that the sequence $\left\{f_{\ell}^{\star}\right\}$ is bounded in $Y$. By contradiction, we assume that $\left\{f_{\ell}^{\star}\right\}$ is not bounded in $Y$, then passing to a subsequence still denoted $\left\{f_{\ell}^{\star}\right\}$, we have

$$
\left\|f_{\ell}^{\star}\right\|_{Y} \longrightarrow+\infty \text { as } \ell \longrightarrow+\infty .
$$

By using the equality (52) and assumption $(17)_{\ell}(\mathrm{b})$, we have

$$
£_{\ell}\left(x_{\ell}^{\star}, f_{\ell}^{\star}\right) \geq \mathcal{D}_{\ell}\left(f_{\ell}^{\star}\right) .
$$

Therefore, passing to the limit as $\ell \longrightarrow \infty$ in this inequality and using (60) combined with assumption (55) we can derive

$$
\lim £_{\ell}\left(x_{\ell}^{\star}, f_{\ell}^{\star}\right)=+\infty .
$$

On the other side, since $\left(x_{\ell}^{\star}, f_{\ell}^{\star}\right)$ represents a solution to (51) for each $\ell \in \mathbb{N}$ we have

$$
£_{\ell}\left(x_{\ell}^{\star}, f_{\ell}^{\star}\right) \leq £_{\ell}\left(x_{\ell}, f_{\ell}\right), \quad \forall\left(x_{\ell}, f_{\ell}\right) \in \mathcal{U}_{a d}^{\ell} .
$$

We now denote by $x_{\ell}^{0}$ the solution of (24) for $f_{\ell}=f$. Then

$$
\left(x_{\ell}^{0}, f\right) \in \mathcal{U}_{a d}^{\ell}
$$

and from (62) and (52), we have

$$
£_{\ell}\left(x_{\ell}^{\star}, f_{\ell}^{\star}\right) \leq \mathcal{P}_{\ell}\left(x_{\ell}^{0}\right)+\mathcal{D}_{\ell}(f) .
$$

From (29)-(30) and Theorem 3, we have

$$
x_{\ell}^{0} \rightarrow x \in X \text { as } \ell \longrightarrow \infty
$$

where $x$ represents the solution of (1). Then, assumptions (53) and (56) imply that

$$
\mathcal{P}_{\ell}\left(x_{\ell}^{0}\right)+\mathcal{D}_{\ell}(f) \rightarrow \mathcal{P}(x)+\mathcal{D}(f) .
$$

Relations (61), (63) and (65) lead to a contradiction, which concludes the claim.

(ii) Convergence analysis. In this step we prove the convergence of (57) and (58). First, since the sequence $\left\{f_{\ell}^{\star}\right\}$ is bounded in $Y$ we can find a subsequence again denoted by $\left\{f_{\ell}^{\star}\right\}$ and an element $f^{\star} \in Y$ such that (57) holds. Next, we denote by $x^{\star}$ the solution of (1) for $f=f^{\star}$. Then, we have

$$
\left(x^{\star}, f^{\star}\right) \in \mathcal{U}_{a d} .
$$

Furthermore, assumption (29), the convergence (57) and Theorem 3 imply that (58) holds as well.

(iii) Optimality of the limit analysis. We now prove that $\left(x^{\star}, f^{\star}\right)$ is a solution to the optimal control (3). We use the convergence (57), (58) and assumptions (53), (54), to see that

$$
\liminf \left(\mathcal{P}_{\ell}\left(x_{\ell}^{\star}\right)+\mathcal{D}_{\ell}\left(f_{\ell}^{\star}\right)\right) \geq \mathcal{P}\left(x^{\star}\right)+\mathcal{D}\left(f^{\star}\right)
$$


and, therefore, the structure (52) and (16) of the functionals $£_{\ell}$ and $£$ shows that

$$
£\left(x^{\star}, f^{\star}\right) \leq \liminf £_{\ell}\left(x_{\ell}^{\star}, f_{\ell}^{\star}\right)
$$

Next, we fix a solution $\left(x_{0}^{\star}, f_{0}^{\star}\right)$ of (3) and, moreover, for each $\ell \in \mathbb{N}$ we denote by $\tilde{x}_{\ell}^{0}$ the solution of (24) for $f_{\ell}=f_{0}^{\star}$. It follows that

$$
\left(\tilde{x}_{\ell}^{0}, f_{0}^{\star}\right) \in \mathcal{U}_{a d}^{\ell}
$$

and from the optimality of the pair $\left(x_{\ell}^{\star}, f_{\ell}^{\star}\right)$, we have

$$
£_{\ell}\left(x_{\ell}^{\star}, f_{\ell}^{\star}\right) \leq £_{\ell}\left(\tilde{x}_{\ell}^{0}, f_{0}^{\star}\right), \quad \forall \ell \in \mathbb{N} .
$$

We proceed to the upper limit of this inequality to discover that

$$
\lim \sup £_{\ell}\left(x_{\ell}^{\star}, f_{\ell}^{\star}\right) \leq \limsup £_{\ell}\left(\tilde{x}_{\ell}^{0}, f_{0}^{\star}\right) .
$$

Now, we know that $x_{0}^{\star}$ is the solution of the inequality (1) for $f=f_{0}^{\star}$ and $\tilde{x}_{\ell}^{0}$ is the solution of the inequality (1) for $f_{\ell}=f_{0}^{\star}$. As a result, the convergence (29) and Theorem 3 imply that

$$
\tilde{x}_{\ell}^{0} \longrightarrow x_{0}^{\star} \in X \text { as } \ell \longrightarrow \infty .
$$

From the assumptions (53) and (56), we have

$$
\mathcal{P}_{\ell}\left(\tilde{x}_{\ell}^{0}\right) \longrightarrow \mathcal{P}_{\ell}\left(x_{0}^{\star}\right), \quad \mathcal{D}_{\ell}\left(f_{0}^{\star}\right) \longrightarrow \mathcal{D}\left(f_{0}^{\star}\right) \text { as } \ell \longrightarrow \infty \text {. }
$$

We now use (52), (69) and (16) to get

$$
\lim £_{\ell}\left(\tilde{x}_{\ell}^{0}, f_{0}^{\star}\right)=\lim £\left(x_{0}^{\star}, f_{0}^{\star}\right) .
$$

Therefore, (67), (68) and (70) imply that

$$
£\left(x^{\star}, f^{\star}\right) \leq £\left(x_{0}^{\star}, f_{0}^{\star}\right) .
$$

On the other side, since $\left(x_{0}^{\star}, f_{0}^{\star}\right)$ is a solution of (3), we have

$$
£\left(x_{0}^{\star}, f_{0}^{\star}\right)=\min _{(x, f) \in \mathcal{U}_{a d}} £(x, f),
$$

and, therefore the inclusion (66) implies that

$$
£\left(x_{0}^{\star}, f_{0}^{\star}\right) \leq £\left(x^{\star}, f^{\star}\right) .
$$

We now combine the inequalities (71) and (73) to see that

$$
£\left(x^{\star}, f^{\star}\right)=£\left(x_{0}^{\star}, f_{0}^{\star}\right) .
$$

Finally, relations (66), (74) and (72) imply that (59) holds and proof is completed.

\section{A frictional Contact Mechanical Problem}

In this section, we describe the mathematical model for the equilibrium of elastic bodies in frictional contact with a foundation. To this end, we need some precise notations and assumptions.

Let $d \in\{2,3\}$. We denote by $\mathbb{S}^{d}$ the space of second order symmetric tensors on $\mathbb{R}^{d}$ and use the notation ". .", $\|\cdot\|$ and $\mathbf{0}$ for the inner product, the norm, and the zero element of the spaces $\mathbb{R}^{d}$ and $\mathbb{S}^{d}$, respectively. Let $\mho \subset \mathbb{R}^{d}$ be a domain with smooth boundary $\partial \mho$ divided into three measurable disjoint parts $\Gamma_{1}, \Gamma_{2}$ and $\Gamma_{3}$ such that meas $\left(\Gamma_{1}\right)>0$. 
A generic point in $\mho \cup \Gamma$ will be denoted by $\mathbf{u}=\left(u_{i}\right)$ and $\mathbf{y}=v_{i}$ represents the unit outward normal to $\Gamma$. We use the standard notation for Sobolev and Lebesgue spaces corresponding to $\mho$ and $\Gamma$. We use the spaces $L^{2}(\mho)^{d}, L^{2}\left(\Gamma_{2}\right)^{d}, L^{2}\left(\Gamma_{3}\right)$ and $H^{1}(\mho)^{d}$ with their canonical inner products and related norms. Furthermore, for an element $\mathbf{y} \in H^{1}(\mho)^{d}$ we continue to write $\mathbf{y}$ for the trace of $\mathbf{y}$ to $\Gamma$. In addition, we consider the space

$$
\mathrm{U}=\left\{\mathbf{y} \in H^{1}(\mho)^{d}: \mathbf{y}=0 \text { on } \Gamma_{1}\right\},
$$

is a real Hilbert space with canonical inner product

$$
\langle\mathbf{x}, \mathbf{y}\rangle_{\mathrm{U}}=\int_{\mho} \varepsilon(\mathbf{x}) \cdot \varepsilon(\mathbf{y}) d u
$$

and the associated norm $\|\cdot\|_{\mathrm{U}}$. The $\varepsilon$ represents the deformation operator, i.e.,

$$
\varepsilon(\mathbf{x})=\left(\varepsilon_{i j}(\mathbf{x})\right), \quad \varepsilon_{i j}(\mathbf{x})=\frac{1}{2}\left(x_{i, j}+x_{j, i}\right),
$$

where an index that follows a comma denotes the partial derivative with respect to the corresponding component of $\mathbf{u}$, e.g.,

$$
x_{i, j}=\frac{\partial x_{i}}{\partial j} .
$$

Since the space $\mathrm{U}$ is completed then the assumption meas $\left(\Gamma_{1}\right)>0$ allows us to apply Korn's inequality. We denote by $0_{U}$ the zero element of $U$ and we recall that, for an element $\mathbf{y} \in \mathrm{U}$, the normal and tangential components on $\Gamma$ are given by

$$
y_{\mathrm{U}}=\mathbf{y} \cdot \mathbf{y} \text { and } \mathbf{y}_{\tau}=\mathbf{y}-y_{\mathrm{U}} \mathbf{y},
$$

respectively. Recall the trace inequality

$$
\|\mathbf{y}\|_{L^{2}(\Gamma)^{d}} \leq d_{0}\|\mathbf{y}\|_{\mathrm{U}}, \forall \mathbf{y} \in \mathbf{U},
$$

where $d_{0}$ is a positive constant.

For the purpose of simplicity, we consider the data $\mathcal{F}, p, \mathbf{f}_{0}, \mathbf{f}_{2}, \mu$ and $\kappa$ which satisfy the following assumptions.

$$
\begin{aligned}
& \left\{\begin{array}{c}
(a) \mathcal{F}: \mathbb{S}^{d} \longrightarrow \mathbb{S}^{d} . \\
(b) \text { There exists } \beta_{\mathcal{F}}>0 \text { such that } \\
\left\|\mathcal{F}\left(\varepsilon_{1}\right)-\mathcal{F}\left(\varepsilon_{2}\right)\right\| \leq \beta_{\mathcal{F}}\left\|\varepsilon_{1}-\varepsilon_{2}\right\|, \quad \forall \varepsilon_{1}, \varepsilon_{2} \in \mathbb{S}^{d} . \\
(c) \text { There exists } \alpha_{\mathcal{F}}>0 \text { such that } \\
\left\langle\mathcal{F}\left(\varepsilon_{1}\right)-\mathcal{F}\left(\varepsilon_{2}\right), \varepsilon_{1}-\varepsilon_{2}\right\rangle \geq \alpha_{\mathcal{F}}\left\|\mathcal{F}\left(\varepsilon_{1}\right)-\mathcal{F}\left(\varepsilon_{2}\right)\right\|^{2} \\
\geq \alpha_{\mathcal{F}} \beta_{\mathcal{F}}^{2}\left\|\varepsilon_{1}-\varepsilon_{2}\right\|^{2}, \forall \varepsilon_{1}, \varepsilon_{2} \in \mathbb{S}^{d} .
\end{array}\right. \\
& \left\{\begin{array}{r}
(a) p: \mathbb{R} \longrightarrow \mathbb{R}_{+} . \\
(b) \text { There exists } \beta_{p}>0 \text { such that } \\
\left|p\left(r_{1}\right)-p\left(r_{2}\right)\right| \leq \beta_{p}\left|r_{1}-r_{2}\right|, \forall r_{1}, r_{2} \in \mathbb{R} . \\
(c)\left\langle p\left(r_{1}\right)-p\left(r_{2}\right), r_{1}-r_{2}\right\rangle \geq 0, \forall r_{1}, r_{2} \in \mathbb{R} . \\
(d) p(r)=0 \Longleftrightarrow r \leq 0 . \\
\mathbf{f}_{0} \in L^{2}(\mho)^{d}, \mathbf{f}_{2} \in L^{2}\left(\Gamma_{2}\right)^{d} . \\
\mu>0 . \\
d_{0}^{2} \mu \beta_{p}<\alpha_{\mathcal{F}} \beta_{\mathcal{F}}^{2} .
\end{array}\right.
\end{aligned}
$$


$\kappa>0$.

Moreover, we use $Y$ for the product space $L^{2}(\mho)^{d} \times L^{2}\left(\Gamma_{3}\right)^{d}$ equipped with the canonical inner product, and $\Omega$ for the set defined by

$$
\Omega=\left\{\mathbf{y} \in \mathrm{U}: y_{v} \leq \kappa \text { a.e. on } \Gamma_{3}\right\}
$$

So there is the inequality of problem in order to find $x \in \Omega$ such that

$$
\begin{aligned}
\int_{\mho} \mathcal{F}(\varepsilon(\mathbf{x})) \cdot(\varepsilon(\mathbf{y})-\varepsilon(\mathbf{x})) d u & +\int_{\Gamma_{3}} p\left(x_{v}\right)\left(y_{v}-x_{v}\right) d a \\
& +\int_{\Gamma_{3}} \mu p\left(x_{v}\right)\left(\left\|\mathbf{y}_{\tau}\right\|-\left\|\mathbf{x}_{\tau}\right\|\right) d a \geq \int_{\mho} \mathbf{f}_{0} \cdot(\mathbf{y}-\mathbf{x}) d u .
\end{aligned}
$$

It can be demonstrated that the problem (84) is a variational formulation of a mathematical model that describes the equilibrium of the elastic body $\mho$, which is acted on by external forces, is fixed on $\Gamma_{1}$, and is in frictional contact with $\Gamma_{3}$. The contact takes place with the rigid body foundation covered by a layer of deformable material of a thickness $\kappa$. The elasticity operator is denoted by $\mathcal{F}$, and the density of applied body forces and traction acting on the body is denoted by $\mathbf{f}_{0}$ and $\mathbf{f}_{2}$ respectively, and surface $\Gamma_{2}$. A function $p$ describes the reaction of the deformable material, and $\mu$ represents the coefficient of friction.

Next, we consider the constants $\sigma_{0}, \sigma_{2}, \sigma_{3}$ and a function $\theta$ such that

$$
\sigma_{0}>0, \quad \sigma_{2}>0, \quad \sigma_{3}>0, \quad \theta \in L^{2}\left(\Gamma_{3}\right)
$$

We associate to (84) the set of admissible pairs $\mathcal{U}_{a d}^{c}$ and the cost functional $£$ given by

$$
\begin{gathered}
\mathcal{U}_{a d}^{c}=\left\{(\mathbf{x}, \mathbf{f}) \in \Omega \times Y \text { such that } \mathbf{f}=\left(\mathbf{f}_{0}, \mathbf{f}_{2}\right) \text { and (84) holds }\right\}, \\
\begin{aligned}
£(\mathbf{x}, \mathbf{f})=\sigma_{0} & \int_{\mho}\left\|\mathbf{f}_{0}\right\|^{2} d u+\sigma_{2} \int_{\Gamma_{2}}\left\|\mathbf{f}_{2}\right\|^{2} d a \\
& +\sigma_{3} \int_{\Gamma_{3}}\left|x_{v}-\theta\right|^{2} d a, \forall \mathbf{u} \in \mathbf{U}, \mathbf{f}=\left(\mathbf{f}_{0}, \mathbf{f}_{2}\right) \in Y .
\end{aligned}
\end{gathered}
$$

Furthermore, we consider the optimal control problem for finding $\left(\mathbf{x}^{\star}, \mathbf{f}^{\star}\right) \in \mathcal{U}_{a d}^{c}$ such that

$$
£\left(\mathbf{x}^{\star}, \mathbf{f}^{\star}\right)=\min _{(\mathbf{x}, \mathbf{f}) \in \mathcal{U}_{a d}^{c}} £(\mathbf{x}, \mathbf{f}) .
$$

Next, we take a look at a function $q$ and a constant $\tilde{\kappa}$ satisfy the following conditions.

$$
\left\{\begin{array}{l}
(a) \quad q: \mathbb{R} \longrightarrow \mathbb{R}_{+} . \\
\text {(b) There exists } \beta_{q}>0 \text { such that } \\
\left|q\left(r_{1}\right)-q\left(r_{2}\right)\right| \leq \beta_{q}\left|r_{1}-r_{2}\right|, \forall r_{1}, r_{2} \in \mathbb{R} . \\
\text { (c) }\left\langle q\left(r_{1}\right)-q\left(r_{2}\right), r_{1}-r_{2}\right\rangle \geq 0, \forall r_{1}, r_{2} \in \mathbb{R} . \\
\text { (d) } q(r)=0 \Longleftrightarrow r \leq 0 . \\
\tilde{\kappa} \geq \kappa>0 .
\end{array}\right.
$$

Now, we define the set

$$
\tilde{\Omega}=\left\{\mathbf{y} \in \mathrm{U}: y_{v} \leq \tilde{\kappa} \text { on } \Gamma_{3}\right\}
$$

and assume that for each $\ell \in \mathbb{N}$, the functions $\mathbf{f}_{0 \ell}, \mathbf{f}_{2 \ell}, \theta_{\ell}$ and $\gamma_{\ell}$ satisfy the following conditions:

$$
\begin{gathered}
\mathbf{f}_{0 \ell} \in L^{2}(\mho \delta)^{d}, \quad \mathbf{f}_{2 \ell} \in L^{2}\left(\Gamma_{3}\right)^{d}, \\
\gamma_{\ell}>0, \quad \theta_{\ell} \in L^{2}\left(\Gamma_{3}\right) .
\end{gathered}
$$


Now, for each $\ell \in \mathbb{N}$, we consider the following perturbation of (84) for finding $\mathbf{x}_{\ell} \in \Omega$ such that

$$
\begin{aligned}
& \int_{\mho} \mathcal{F}\left(\varepsilon\left(\mathbf{x}_{\ell}\right)\right) \cdot\left(\varepsilon(\mathbf{y})-\varepsilon\left(\mathbf{x}_{\ell}\right)\right) d u+\int_{\Gamma_{3}} p\left(x_{\ell v}\right)\left(y_{v}-x_{\ell v}\right) d a \\
& +\frac{1}{\gamma_{\ell}} \int_{\Gamma_{3}} q\left(x_{\ell v}-\kappa\right)\left(y_{v}-x_{\ell v}\right) d a+\mu \int_{\Gamma_{3}} p\left(x_{\ell v}\right)\left(\left\|\mathbf{y}_{\tau}\right\|-\left\|\mathbf{x}_{\ell \tau}\right\|\right) d a \\
& \geq \int_{\mho} \mathbf{f}_{0 \ell} \cdot(\mathbf{y}-\mathbf{x}) d u+\int_{\Gamma_{2}} \mathbf{f}_{2 \ell} \cdot\left(\mathbf{y}-\mathbf{x}_{\ell}\right) d a \forall \mathbf{y} \in \tilde{\Omega} .
\end{aligned}
$$

The equation (94) denotes the variational formulation of the contact problem, where the foundation of a rigid body is covered by a layer of deformable material of thickness $\tilde{\kappa}$. This layer is divided into two parts: a first layer of $\tilde{\kappa}-\kappa>0$ thickness located on the top of the rigid body, and a second layer of $\kappa$ thickness located above. The $\gamma_{\ell}$ is a deformability coefficient of the first layer and, $\frac{1}{\gamma_{\ell}}$ denotes its stiffness coefficient. In addition, $q$ is a normal compliance function that describes the reaction of the first layer and the foundation, which is denoted by $\mathcal{F}_{\tilde{\kappa}}$.

We associate to (94), the set of admissible pairs $\mathcal{U}_{a d}^{c \ell}$ and the cost function $£_{\ell}$ given by

$$
\begin{gathered}
\mathcal{U}_{a d}^{c \ell}=\left\{\left(\mathbf{x}_{\ell}, \mathbf{f}_{\ell}\right) \in \tilde{\Omega} \times Y \text { such that } \mathbf{f}=\left(\mathbf{f}_{0 \ell}, \mathbf{f}_{2 \ell}\right) \text { and (94) holds }\right\} \\
£_{\ell}\left(\mathbf{x}_{\ell}, \mathbf{f}_{\ell}\right)=\sigma_{0} \int_{\mho}\left\|\mathbf{f}_{0 \ell}\right\|^{2} d u+\sigma_{2} \int_{\Gamma_{2}}\left\|\mathbf{f}_{2 \ell}\right\|^{2} d a+\sigma_{3} \int_{\Gamma_{3}}\left|x_{\ell v}-\theta_{\ell}\right|^{2} d a, \\
\forall \mathbf{x}_{\ell} \in \mathrm{U}, \mathbf{f}_{\ell}=\left(\mathbf{f}_{0 \ell}, \mathbf{f}_{2 \ell}\right) \in Y .
\end{gathered}
$$

Theorem 5. Assume that (77)-(82), (85), (89)-(90), (92) and (93) hold. Then

(a) the problem (84) has a unique solution and, for each $\ell \in \mathbb{N}$, problem (94) has a unique solution. Moreover, if

$$
\gamma_{\ell} \longrightarrow 0, \mathbf{f}_{0 \ell} \rightarrow \mathbf{f}_{0} \in L^{2}(\mho), \mathbf{f}_{2 \ell} \rightarrow \mathbf{f}_{2} \in L^{2}\left(\Gamma_{3}\right) \text { as } \ell \longrightarrow \infty,
$$

the solution of problem (94) converges to the solution of problem (84), i.e.,

$$
\mathbf{x}_{\ell} \rightarrow \mathbf{x} \in U \text { and } \mathbf{u}_{\ell} \rightarrow \mathbf{u} \subset U \text { as } \ell \longrightarrow \infty
$$

(b) the problem (88) has at least one solution and the problem (96) has at least one solution for each $\ell \in \mathbb{N}$. Moreover, if

$$
\gamma_{\ell} \longrightarrow 0, \theta_{\ell} \longrightarrow \theta \in L^{2}\left(\Gamma_{3}\right) \text {, as } \ell \longrightarrow \infty,
$$

and $\left\{\left(\mathbf{x}_{\ell}^{\star}, \mathbf{f}_{\ell}^{\star}\right)\right\}$ is a sequence of solutions of problem (96), there exists a subsequence of the sequence $\left\{\left(\mathbf{x}_{\ell}^{\star}, \mathbf{f}_{\ell}^{\star}\right)\right\}$, again denoted by $\left\{\left(\mathbf{x}_{\ell}^{\star}, \mathbf{f}_{\ell}^{\star}\right)\right\}$, and a solution $\left(\mathbf{x}^{\star}, \mathbf{f}^{\star}\right)$ of the problem (88), such that

$$
\mathbf{f}_{\ell}^{\star} \rightarrow \mathbf{f}^{\star} \in Y, \mathbf{x}_{\ell}^{\star} \rightarrow \mathbf{x}^{\star} \in \mathrm{U} \text { as } \ell \longrightarrow \infty
$$

Proof. First, we denote by $\varsigma: U \longrightarrow Y$ the operator, $\mathbf{y} \longmapsto\left\langle\iota \mathbf{y}, \zeta_{2} \mathbf{y}\right\rangle$, where $\iota: U \longrightarrow L^{2}(\mho)^{d}$ is the canonic embedding and $\zeta_{2}: U \longrightarrow L^{2}\left(\Gamma_{2}\right)^{d}$ is the restriction to the trace map to $\Gamma_{2}$. Next, we take the operators $\mathcal{N}: U \longrightarrow U, \mathcal{M}: U \longrightarrow U, \mathcal{T}: U \longrightarrow 2^{\mathrm{U}}$, the function

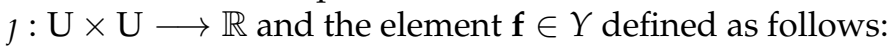

$$
\begin{gathered}
\langle\mathcal{N}(\mathbf{u}), \mathbf{y}\rangle_{\mathrm{U}}=\int_{\mho} \mathcal{F}(\varepsilon(\mathbf{x})) \cdot \varepsilon(\mathbf{y}) d u+\int_{\Gamma_{3}} p\left(x_{v}\right) y_{v} d a, \quad \forall \mathbf{u} \in \mathcal{T}(\mathbf{x}), \\
\langle\mathcal{M}(\mathbf{u}), \mathbf{y}\rangle_{\mathrm{U}}=\int_{\urcorner_{3}} q\left(x_{v}-\kappa\right) y_{v} d a, \quad \forall \mathbf{u} \in \mathcal{T}(\mathbf{x}),
\end{gathered}
$$




$$
\begin{gathered}
\jmath: \mathbf{U} \times \mathbf{U} \longrightarrow \mathbb{R}, \quad \jmath(\mathbf{x}, \mathbf{y})=\mu \int_{\Gamma_{3}} p\left(x_{v}\right)\left\|\mathbf{y}_{\tau}\right\| d a, \\
\mathbf{f}=\left(\mathbf{f}_{0}, \mathbf{f}_{2}\right), \quad \forall \mathbf{x}, \mathbf{y} \in \mathbf{U} .
\end{gathered}
$$

Then it is clear that

$\mathbf{x} \in \Omega, \mathbf{u} \in \mathcal{T}(\mathbf{x})$ is a solution of problem (84) if and only if

$$
\langle\mathcal{N}(\mathbf{u}), \mathbf{y}-\mathbf{x}\rangle_{\mathrm{U}}+\jmath(\mathbf{x}, \mathbf{y})-\jmath(\mathbf{x}, \mathbf{x}) \geq\langle\mathbf{f}, \varsigma \mathbf{y}-\varsigma \mathbf{x}\rangle_{Y}, \forall y \in \Omega .
$$

For each $\ell \in \mathbb{N}, \mathbf{x}_{\ell} \in \tilde{\Omega}, \mathbf{u}_{\ell} \in \mathcal{T}\left(\mathbf{x}_{\ell}\right)$ is a solution of problem (94) if and only if

$$
\begin{aligned}
\left\langle\mathcal{N}\left(\mathbf{u}_{\ell}\right), \mathbf{y}-\mathbf{x}_{\ell}\right\rangle_{\mathrm{U}}+\frac{1}{\gamma_{\ell}}\left\langle\mathcal{M}\left(\mathbf{u}_{\ell}\right), \mathbf{y}-\mathbf{x}_{\ell}\right\rangle_{\mathrm{U}} & +\jmath\left(\mathbf{x}_{\ell}, \mathbf{y}\right)-\jmath\left(\mathbf{x}_{\ell}, \mathbf{x}_{\ell}\right) \\
& \geq\left\langle\mathbf{f}_{\ell}, \mathbf{y}-\mathbf{x}_{\ell}\right\rangle_{\Upsilon}, \forall \mathbf{y} \in \tilde{\Omega} .
\end{aligned}
$$

We can now continue with the proof of the two parts of the theorem.

(a) We deploy the abstract results in Sections 2 and 3 with $X=U, Y=L^{2}(\mho)^{d} \times L^{2}\left(\Gamma_{2}\right)^{d}$, $\Omega$ and $\tilde{\Omega}$ defined by (83) and (91), respectively. The operators $\mathcal{N}$ and $\mathcal{T}$ defined by (101), $\mathcal{M}$ defined by (102), $\boldsymbol{j}$ defined by (103) and $\mathbf{f}$ given by (104). It is easy to see that the assumptions (7)-(15) and (25)-(32) are held.

Using the assumption (77), we have

$$
\begin{aligned}
\langle\mathcal{N}(\mathbf{u})-\mathcal{N}(\mathbf{v}), \mathbf{x}-\mathbf{y}\rangle_{\mathrm{U}} & \geq \alpha_{\mathcal{F}} \beta_{\mathcal{F}}^{2}\|\mathbf{x}-\mathbf{y}\|_{\mathrm{U}}^{2}, \forall \mathbf{u} \in \mathcal{T}(\mathbf{x}), \mathbf{v} \in \mathcal{T}(\mathbf{y}) \\
\|\mathcal{N}(\mathbf{u})-\mathcal{N}(\mathbf{v})\| & \leq\left(\beta_{\mathcal{F}}+d_{0}^{2} \beta_{p}\right)\|\mathbf{x}-\mathbf{y}\|_{\mathrm{U}}, \quad \forall \mathbf{x}, \mathbf{y} \in \mathrm{U}, \mathbf{u} \in \mathcal{T}(\mathbf{x}), \mathbf{v} \in \mathcal{T}(\mathbf{y}) .
\end{aligned}
$$

Hence, from conditions (8)-(9) hold with

$$
\alpha_{\mathcal{N}} \beta_{\mathcal{N}}^{2} \alpha_{\mathcal{T}}^{2}=\alpha_{\mathcal{F}} \beta_{\mathcal{F}}^{2}
$$

The assumption (11) obviously holds; and on the other side, an elementary evaluation based on the definition (103) and the trace inequality (76) shows that

$$
\begin{gathered}
\jmath\left(\mathbf{x}_{1}, \mathbf{y}_{2}\right)-\jmath\left(\mathbf{x}_{1}, \mathbf{y}_{2}\right)+\jmath\left(\mathbf{x}_{2}, \mathbf{y}_{1}\right)-\jmath\left(\mathbf{x}_{2}, \mathbf{y}_{2}\right) \leq d_{0}^{2} \mu \beta_{p}\left\|\mathbf{x}_{1}-\mathbf{x}_{2}\right\|_{\mathrm{U}}\left\|\mathbf{y}_{1}-\mathbf{y}_{2}\right\|_{\mathrm{U}} \\
\text { for all } \mathbf{x}_{1}, \mathbf{x}_{2}, \mathbf{y}_{1}, \mathbf{y}_{2} \in \mathrm{U} .
\end{gathered}
$$

Now, if $\alpha_{j}=d_{0}^{2} \mu \beta_{p}$, the assumption (12) holds true, and if $\rho=0$ and $\varrho=d_{0}^{2} \mu \beta_{p}$, the assumption (22) also holds true. Using (81), it satisfies the smallness conditions (13) and (20). We also note that conditions (21)-(21) arise from standard compactness arguments and, finally, assumption (32) is a direct consequence of the definitions (102), (91) and (83), combined with (89) of the function $q$.

Therefore we deploy the Theorem 1 and Lemma 1 to derive the existence of a unique solution of the problems (105) and (106), respectively. Furthermore, when (97) holds, we can derive the convergence (98) from Theorem 3 . These results, combined with (105) and (106) allow us to arrive at the proof of the first part of the theorem.

(b) Next, we apply the abstract results of Sections 2 and 4 in the framework of functional $£$ and $\ell_{\ell}$ defined in (87) and (96), respectively. It is clear to see that in this case the conditions (7)-(13), (15)-(22), (25)-(28), (17), (18) $)_{\ell}$ and (52)-(56) hold with suitable choice of functions $\mathcal{P}, \mathcal{D}, \mathcal{P}_{\ell}$ and $\mathcal{D}_{\ell}$. Obviously, we are in a position to use the Lemma 2 to derive the existence of a solution of the optimal control problems in (88) and (96). The convergence (100) is proved by utilizing the Theorem 4 . 


\section{A Stationary Heat Transfer Boundary Value Problem with Unilateral Constraints}

This section is devoted to the study of the mathematical model that describes a heat transfer boundary value problem. The classical formulation of the model is: Finding a temperature field $x: \mho \longrightarrow \mathbb{R}$ such that

$$
\left\{\begin{array}{l}
\text { (a) } x \geq 0, \Delta x+f \leq 0, x(\Delta x+f)=0 \quad \text { a.e. in } \mho, \\
\text { (b) } x=0, \text { a.e. in } \Gamma_{1}, \\
\text { (c) } x=b, \text { a.e. in } \Gamma_{2}, \\
\text { (d) }-\frac{\partial x}{\partial v}=q, \text { a.e. in } \Gamma_{3} .
\end{array}\right.
$$

Here, $\Omega$ is a bounded domain in $\mathbb{R}^{d}(d=1,2,3)$ with smooth boundary $\partial \mho \mho=\Gamma_{1} \cup \Gamma_{2} \cup \Gamma_{3}$ and outer normal unit $\mathbf{y}$. Assume that $\Gamma_{1}, \Gamma_{2}, \Gamma_{3}$ are disjoint measurable sets and meas $\left(\Gamma_{1}\right)>$ 0 . Furthermore, we leave out the dependence of the different functions on the spatial variable $\mathbf{x} \in \mho \cup \partial \mho$ in (109). The function $f$ represents the internal energy, $b$ is the prescribed temperature field on $\Gamma_{2}$ and $q$ represents the heat flux on $\Gamma_{3}$. Furthermore, the normal derivative of $x$ on $\Gamma_{3}$ is denoted by $\frac{\partial x}{\partial v}$.

For the variational formulation of (109), we consider the space

$$
\mathrm{U}=\left\{\mathbf{y} \in H^{1}(\mho): \mathbf{y}=0 \text { on } \Gamma_{1}\right\} .
$$

Let $\left(\mathrm{U},\langle\cdot, \cdot\rangle_{\mathrm{U}}\right)$ be a real Hilbert space. Assume that

$$
f \in L^{2}(\mho), \quad b \in L^{2}\left(\Gamma_{2}\right), q \in L^{2}\left(\Gamma_{3}\right),
$$

there exists $y_{0} \in \mathrm{U}$ such that

$$
0 \leq y_{0} \in \mho \text { and } y_{0}=b \text { on } \Gamma_{2}
$$

We begin by introducing the set

$$
\Omega=\left\{y \in \mathrm{U}: y \geq 0 \text { on } \mho, y=b \text { on } \Gamma_{2}\right\}
$$

We note that the assumption (111) represents a compatibility assumption on $b$ which shows that the set $\Omega$ is not empty. Then, the variational formulation of the equation (109), to be achieved as: For finding $x \in \Omega$ such that

$$
\int_{\mho} \nabla x \cdot(\nabla y-\nabla x) d u+\int_{\Gamma_{3}} q(y-x) d a \geq \int_{\mho} f(y-x) d u, \forall y \in \Omega .
$$

Now, we introduce the set of admissible pairs for inequality (113) defined by

$$
\mathcal{U}_{\text {ad }}^{t}=\left\{(x, f) \in \Omega \times L^{2}(\mho) \text { such that (113) holds }\right\} .
$$

Moreover, we consider two constants $\omega, \delta$ and a function $\psi$ such that

$$
\omega>0, \delta>0, \quad \psi \in L^{2}(\delta) .
$$

Now we associate to (113) the above data, for finding the optimal control problem $\left(x^{\star}, f^{\star}\right) \in$ $\mathcal{U}_{a d}^{t}$ such that

$$
\omega \int_{\mho}\left(x^{\star}-\psi\right)^{2} d u+\delta \int_{\mho}\left(f^{\star}\right)^{2} d u=\min _{(x, f) \in \mathcal{U}_{a d}^{t}}\left\{\omega \int_{\mho}(x-\psi)^{2} d u+\delta \int_{\mho} f^{2} d u\right\} .
$$

Next, we introduce the set

$$
\tilde{\Omega}=\{y \in \mathrm{U}: y \geq 0 \text { in } \mho\}
$$


For each $\ell \in \mathbb{N}$, we assume that the functions $f_{\ell}, \psi_{\ell}$ and the constants $\gamma_{\ell}, \omega_{n}, \delta_{\ell}$, are given and satisfy the following conditions:

$$
\begin{gathered}
f_{\ell} \in L^{2}(\mho), \\
\gamma_{\ell}>0, \omega_{\ell}>0, \quad \delta_{\ell}>0, \quad \psi_{\ell} \in L^{2}(\mho) .
\end{gathered}
$$

Then, for each $\ell \in \mathbb{N}$, we consider the following perturbation of (113) for finding $x_{\ell} \in \tilde{\Omega}$ such that

$$
\begin{aligned}
\int_{\mho} \nabla x_{\ell} \cdot\left(\nabla y-\nabla x_{\ell}\right) d u+\int_{\Gamma_{3}} q\left(y-x_{\ell}\right) d a & +\frac{1}{\gamma_{\ell}} \int_{\tau_{2}}\left(x_{\ell}-b\right)\left(y-x_{\ell}\right) d a \\
& \geq \int_{\mho} f_{\ell}\left(y-x_{\ell}\right) d u, \quad \forall y \in \tilde{\Omega} .
\end{aligned}
$$

It is clear that (120) represents the variational formulation of the following boundary value problem for finding a temperature field $x_{\ell}: \mho \longrightarrow \mathbb{R}$ such that

$$
\begin{gathered}
x_{\ell} \geq 0, \Delta x_{\ell}+f_{\ell} \leq 0, \quad x_{\ell}\left(\Delta x_{\ell}+f_{\ell}\right)=0 \text { a.e. in } \mho, \\
\left.x_{\ell}=0 \text { a.e. on }\right\urcorner_{1}, \\
\left.-\frac{\partial x_{\ell}}{\partial v}=\frac{1}{\gamma_{\ell}}\left(x_{\ell}-b\right) \text { a.e. on }\right\urcorner_{2}, \\
\left.-\frac{\partial x_{\ell}}{\partial v}=q \text { a.e. on }\right\urcorner_{3},
\end{gathered}
$$

The set of admissible pairs for inequality (120) is defined by

$$
\mathcal{U}_{a d}^{t \ell}=\left\{\left(x_{\ell}, f_{\ell}\right) \in \tilde{\Omega} \times L^{2}(\mho) \text { such that (120) holds }\right\} .
$$

Furthermore, the associated optimal control problem for finding $\left(x_{\ell}^{\star}, f_{\ell}^{\star}\right) \in \mathcal{U}_{a d}^{t \ell}$ such that

$$
\omega_{\ell} \int_{\mho}\left(x_{\ell}^{\star}-\psi_{\ell}\right)^{2} d u+\delta_{\ell} \int_{\mho}\left(f_{\ell}^{\star}\right)^{2} d u=\min _{(x, f) \in \mathcal{U}_{a d}^{+\ell}}\left\{\omega_{\ell} \int_{\mho}\left(x-\psi_{\ell}\right)^{2} d u+\delta_{\ell} \int_{\mho} f^{2} d u\right\} .
$$

Theorem 6. Assume that (110)-(111), (115), (118) and (119) hold. Then

(a) problem (113) has a unique solution and, for each $\ell \in \mathbb{N}$ the problem (120) has a unique solution also. Furthermore, if

$$
\gamma_{\ell} \longrightarrow 0 \text { and } f_{\ell} \rightarrow f \in L^{2}(\mho) \text { as } \ell \longrightarrow \infty,
$$

the solution of problem (120) converges to the solution of problem (113), i.e.,

$$
x_{\ell} \longrightarrow x \in \mathrm{U} \text { as } \ell \longrightarrow \infty .
$$

(b) problem (116) has at least one solution and, for each $\ell \in \mathbb{N}$, problem (126) has at least one solution also. Moreover, the solution of problem (116) is unique if

$$
\psi=0_{L^{2}(\mho)}
$$

and, for each $\ell \in \mathbb{N}$, the solution of (126) is unique, if

$$
\psi_{\ell}=0_{L^{2}(\mho)} .
$$

(c) Assume that

$$
\gamma_{\ell} \rightarrow 0, \omega_{\ell} \rightarrow \omega, \delta_{\ell} \rightarrow \delta, \quad \psi_{\ell} \rightarrow \psi \in L^{2}(\mho) \text { as } \ell \longrightarrow \infty,
$$


and let $\left\{\left(x_{\ell}^{\star}, f_{\ell}^{\star}\right)\right\}$ be a sequence of solutions of (126). Then, there exists a subsequence of the sequence $\left\{\left(x_{\ell}^{\star}, f_{\ell}^{\star}\right)\right\}$, again denoted by $\left\{\left(x_{\ell}^{\star}, f_{\ell}^{\star}\right)\right\}$, and a solution $\left(x^{\star}, f^{\star}\right)$ of $(116)$, such that

$$
f_{\ell}^{\star} \rightarrow f^{\star} \in L^{2}(\mho), x_{\ell}^{\star} \rightarrow x^{\star} \in \mathrm{U} \text { as } \ell \longrightarrow \infty .
$$

Moreover, if $\psi=0_{L^{2}(\mho)}$, then the entire sequence $\left\{\left(x_{\ell}^{\star}, f_{\ell}^{\star}\right)\right\}$ satisfies (130) where $\left(x^{\star}, f^{\star}\right)$ represents the unique solution of (116).

Proof. To begin, we will introduce a notation that allows us to write the problems in equivalent form. To this end, we denote by $\varsigma: U \longrightarrow L^{2}(\mho)$ the canonical inclusion of $\mathrm{U}$ in $L^{2}(\mho)$. Furthermore, we consider the operators $\mathcal{N}: \mathrm{U} \longrightarrow \mathrm{U}, \mathcal{T}: \mathrm{U} \longrightarrow 2^{\mathrm{U}}$ and $\mathcal{M}: \mathrm{U} \longrightarrow \mathrm{U}$ defined by

$$
\begin{gathered}
\langle\mathcal{N}(u), y\rangle_{\mathrm{U}}=\int_{\mho} \nabla x \cdot \nabla y d u+\int_{\Gamma_{3}} q y d a, \forall x, y \in \mathrm{U}, u \in \mathcal{T}(x), \\
\langle\mathcal{M}(u), y\rangle_{\mathrm{U}}=\int_{\Gamma_{2}}(x-b) y d a, \quad \forall x, y \in \mathrm{U}, u \in \mathcal{T}(x) .
\end{gathered}
$$

Then, it is easy to see that

$$
\left\{\begin{array}{l}
x \text { is a solution of (113) if and only if } \\
x \in \Omega,\langle\mathcal{N}(u), y-x\rangle_{\mathrm{U}} \geq\langle f, y-x\rangle_{L^{2}(\mho)}, \quad \forall y \in \Omega, u \in \mathcal{T}(x) .
\end{array}\right.
$$

Moreover, for each $\ell \in \mathbb{N}$,

$$
\left\{\begin{array}{l}
x_{\ell} \in \tilde{\Omega} \text { is a solution of (120) if and only if } \\
\langle\mathcal{N}(u), y-x\rangle_{\mathrm{U}}+\frac{1}{\gamma_{\ell}}\left\langle\mathcal{M}\left(u_{\ell}\right), y-x_{\ell}\right\rangle_{\mathrm{U}} \geq\left\langle f_{\ell}, y-x_{\ell}\right\rangle_{L^{2}(\mho)}, \forall y \in \tilde{\Omega}, u \in \mathcal{T}(x) .
\end{array}\right.
$$

Next, denote by $£: U \times L^{2}(\mho) \longrightarrow \mathbb{R}$ and $£_{\ell}: U \times L^{2}(\mho) \longrightarrow \mathbb{R}$ the cost functionals given by

$$
\begin{gathered}
£(x, f)=\omega\|x-\psi\|_{L^{2}(\mho)}^{2}+\delta\|f\|_{L^{2}(\mho)^{\prime}}^{2} \\
£_{\ell}(x, f)=\omega_{\ell}\left\|x-\psi_{\ell}\right\|_{L^{2}(\mho)}^{2}+\delta_{\ell}\|f\|_{L^{2}(\mho)^{\prime}}^{2}, \quad \forall(x, f) \in \mathrm{U} \times L^{2}(\mho) .
\end{gathered}
$$

Then, it is clear to see that

$$
\left\{\begin{array}{l}
\left(x^{\star}, f^{\star}\right) \text { is a solution of }(116) \text { if and only if } \\
\left(x^{\star}, f^{\star}\right) \in \mathcal{U}_{a d}^{t} \text { and } £\left(x^{\star}, f^{\star}\right)=\min _{\left(x^{\star}, f^{\star}\right) \in \mathcal{U}_{a d}^{t}} £(x, f) .
\end{array}\right.
$$

Furthermore, for each $\ell \in \mathbb{N}$,

$$
\left\{\begin{array}{l}
\left(x_{\ell}^{\star}, f_{\ell}^{\star}\right) \text { is a solution of (116) if and only if } \\
\left(x_{\ell}^{\star}, f_{\ell}^{\star}\right) \in \mathcal{U}_{a d}^{\ell \ell} \text { and } £_{\ell}\left(x_{\ell}^{\star}, f_{\ell}^{\star}\right)=\min _{\left(x^{\star}, f^{\star}\right) \in \mathcal{U}_{a d}^{t \ell}} £_{\ell}(x, f) .
\end{array}\right.
$$

We now proceed with the proof of the two parts of the theorem.

(a) We use the abstract results from Sections 2 and 3 with $X=U, Y=L^{2}(\mho)$, and the equations (112), (117), (131), (132) and $\jmath \equiv 0$. It is clear to see that in this case, conditions (7)-(15), (25)-(32) hold. Therefore, we use the Theorem 1 and Lemma 1 to derive the existence of a unique solution to the variational inequality problems (133) and (134), respectively. By the Theorem 3, we deduce the convergence result (128). Adding the results (133) and (134), we arrive at the conclusion of the proof of Theorem 6(a).

(b) We use the abstract results from Sections 2 and 4 in the functional framework of (135) and (136), respectively. It is clear to see that in this case, conditions (7)-(13), (15)-(22), 
(25)-(28), (17) $)_{\ell},(18)_{\ell},(52)-(54)$ hold, with suitable choice of the functions $\mathcal{P}, \mathcal{P}_{\ell}, \mathcal{D}$ and $\mathcal{D}_{\ell}$. Therefore, from the Theorem 2 and Lemma 2, we deduce the existence of a solution of the optimal control problems (137) and (138), respectively. The uniqueness of the solution of (116) in the case $\psi=0_{L^{2}(\mho)}$ follows from a strict convexity argument. For any $f \in L^{2}(\mho)$, let $x(f)$ denote the solution of the variational inequality (133). Then it was proved in [32], that the functional

$$
f \longmapsto £(x(f), f)=\omega\|x(f)\|_{L^{2}(\mho)}^{2}+\delta\|f\|_{L^{2}(\mho)}^{2}
$$

is strictly convex. Hence, the optimal control problem (137) has a unique solution. The uniqueness of the solution of (126) in the case $\psi_{\ell}=0_{L^{2}(\mho)}$ follows from the same argument. Adding the results (137) and (138), we arrive at the conclusion of the proof of Theorem 6(b).

Author Contributions: Formal analysis, S.-S.C.; Methodology, S.; Software, L.W.; Writing-original draft, J.T. and L.Z. All authors contributed equally to the writing of this paper. All authors have read and agreed to the published version of the manuscript.

Funding: This work was supported by the Natural Science Foundation of China (Grant 12161088). This work was also supported by the Natural Science Foundation of China Medical University, Taichung,Taiwan and supported by the Scientic Research Fund of Yibin University (2021YY03) .

Institutional Review Board Statement: Not applicable.

Informed Consent Statement: Not applicable.

Data Availability Statement: The data sets used and/or analysed during the current study are available from the corresponding author on reasonable request.

Acknowledgments: The authors wish to express their gratitude to the editor and referees for careful reading of the manuscript, and their valuable comments and suggestions.

Conflicts of Interest: The authors declare that they have no conflict of interest to this work. We declare that we do not have any commercial or associative interest that represents a conflict of interest in connection with that work submitted.

\section{References}

1. Borwein, J.M.; Zhu, Q.J. Techniques of variational analysis. In CMS Books in Mathematics; Springer: New York, NY, USA, 2005; Volume 20.

2. Goeleven, D. Complementarity and Variational Inequalities in Electronics; Academic Press: Cambridge, MA, USA, 2017.

3. Jofre, A.; Rockafellar, R.T.; Wets, R.J.B. Variational Inequalities and Economic Equilibrium. Math. Oper. Resea. 2007, 32, 32-50. [CrossRef]

4. Kikuchi, N.; Oden, J.T. Contact Problems in Elasticity: A Study of Variational Inequalities and Finite Element Methods; SIAM: Philadelphia, PA, USA, 1988.

5. Zeng, B.; Migorski, S. Variational-hemivariational inverse problems for unilateral frictional contact. Appl. Anal. 2020, 99, $293-312$. [CrossRef]

6. Motreanu, D.; Sofonea, M. Quasi variational inequalities and applications in frictional contact problems with normal compliance. Adv. Math. Sci. Appl. 2000, 10, 103-118.

7. Clarke, F.H. Optimization and Nonsmooth Analysis; Wiley: New York, NY, USA, 1983.

8. Brezis, H. Functional analysis, Sobolev spaces and partial differential equations. In Universitext; Springer: New York, NY, USA, 2011.

9. Mordukhovich, B.S. Variational Analysis and Generalized Differentiation. Autom. Remote Control. 2006, 70, $1086-1087$.

10. Salahuddin. Regularization techniques for Inverse variational inequalities involving relaxed cocoercive mapping in Hilbert spaces. Nonlinear Anal. Forum 2014, 19, 65-76.

11. Kim, J.K.; Alesemi, M.; Salahuddin. Convergence theorem of relaxed quasimonotone variational inequality problems. J. Nonlinear Convex Anal. 2021, 22, 2671-2678.

12. Barbu, V. Optimal Control of Variational Inequalities; Research Notes in Mathematics 100 Pitman: Boston, MA, USA, 1984. [CrossRef]

13. Bonnans, J.F.; Tiba, D. Pontryagin's principle in the control of semilinear elliptic variational inequalities. Appl. Math. Optim. 1991, 23, 299-312. [CrossRef]

14. Freidman, A. Optimal control for variational inequalities. SIAM J. Control Optim. 1986, 24, 439-451. [CrossRef] 
15. Mignot, F.; Puel, J.-P. Optimal control in some variational inequalities. SIAM J. Control Optim. 1984, 22, 466-476. 10.1137/0322028. [CrossRef]

16. Peng, Z.; Kunisch, K. Optimal control of elliptic variational-hemivriational inequalities. J. Optim. Theory Appl. 2018, 178, 1-25. [CrossRef]

17. Matei, A.; Micu, S. Boundary optimal control for nonlinear antiplane problems. Nonlinear Anal. Theory Methods Appl. 2011, 74, 1641-1652. [CrossRef]

18. Capatina, A. Variational Inequalities Frictional Contact Problems. In Advances in Mechanics and Mathematics; Springer: New York, NY, USA, 2014; Volume 31.

19. Eck, C.; Jarusek, J.; Krbec, M. Unilateral Contact Problems: Variational Methods and Existence Theorems. In Pure and Applied Mathematics; Chapman/CRC Press: New York, NY, USA, 2005; Volume 270.

20. Boukrouche, M.; Tarzia, D.A. Convergence of distributed optimal control problems governed by elliptic variational inequalities. Comput. Optim. Appl. 2012, 53, 375-393. [CrossRef]

21. Capatina, A. Optimal control of Signorini problem. Numer. Funct. Anal. Optim. 2000, 21, 817-828. 0008816987. [CrossRef]

22. Matei, A.; Micu, S.; Nita, C. Optimal control for antiplane frictional contact problems involving nonlinearly elastic materials of Hencky type. Math. Mech. Solids 2018, 23, 308-328. [CrossRef]

23. Sofonea, M.; Xiao, Y.B. Boundary optimal control of a nonsmooth frictionless contact problem. Comput. Math. Appl. 2019, 78, 152-165. [CrossRef]

24. Noor, M.A.; Noor, K.I.; Rassias, M.T. New Trends in General Variational Inequalities. Acta Appl. Math. 2020, 170, 981-1064. [CrossRef]

25. Thong, D.V.; Cholamjiak, P.; Rassias, M.T.; Cho, Y.J. Strong convergence of inertial subgradient extragradient algorithm for solving pseudomonotone equilibrium problems. Optim. Lett. 2021, 16, 545-573. [CrossRef]

26. Sofonea, M. Optimal control of variational inequalities with applications to contact mechanics. In Current Trends in Mathematical Analysis and Its Interdisciplinary Applications; Dutta, H., Ed.; Springer Nature: Basel, Switzerland, 2019; Chapter 13; pp. $443-487$.

27. Sofonea, M.; Matei, A. Mathematical Models in Contact Mechanics; London Mathematical Society Lecture Note Series; Cambridge University Press:Cambridge, UK, 2012; , Volume 398.

28. Sofonea, M.; Tarzia, D.A. Convergence results for optimal control problems governed by elliptic quasivariational inequalities. Num. Funct. Anal. Optim. 2020, 41, 1326-1351. [CrossRef]

29. Sofonea, M.; Migorski, S. Variational-hemivariational inequalities with applications. In Pure and Applied Mathematics; Chapman and Hall/CRC Press: Boca Raton, FL, USA, 2018.

30. Salahuddin. Penalty Method for Non-Stationary General Variational Like Inequalities. J. Appl. Nonlinear Dyn. 2021, 10, 369-380. [CrossRef]

31. Lee, B.S. Salahuddin: Solutions for general class of hemivariational like inequality systems. J. Nonlinear Convex Anal. 2015, 16, 141-150.

32. Boukrouche, M.; Tarzia, D.A. Existence, uniqueness and convergence of optimal control problems associated with parabolic variational inequalities of the second kind. Nonlinear Anal. Real World Appl. 2011, 12, 2211-2224. 2011.01.003. [CrossRef] 